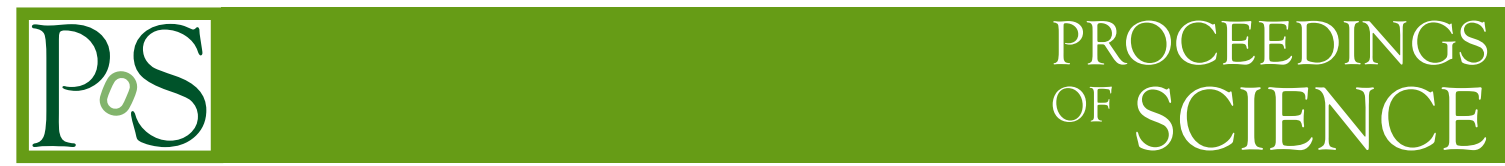

\title{
Antiparticle Detection in Space for Dark Matter Search: the PAMELA Experiment
}

\author{
Oscar Adriani, on behalf of the PAMELA collaboration* \\ INFN Firenze and University of Florence \\ E-mail: adrianiefi.infn.it
}

The PAMELA experiment on satellite is continuously taking data since July 2006, detecting several cosmic ray species, in the energy range from tens of $\mathrm{MeV}$ to hundreds of $\mathrm{GeV}$. In this paper we will present results of the most recent data analyses involving observations of interest for indirect dark-matter searches.

35th International Conference of High Energy Physics

July 22-28, 2010

Paris, France

${ }^{*}$ Speaker. 


\section{Introduction and short description of the apparatus}

PAMELA [1] is a satellite-borne experiment to study antiparticles in the cosmic rays. It has been designed to obtain an unprecedented precision in the measurement of the energy spectra of antiprotons between $80 \mathrm{MeV}$ and $180 \mathrm{GeV}$, positrons in the range $50 \mathrm{MeV}-270 \mathrm{GeV}$, and to obtain high-precision spectra of protons, light nuclei $(\mathrm{Z}<8)$ and electrons. The main purposes of these measurements are to test cosmic-ray propagation models and to search for evidence of dark-matter particles annihilations or other unknown primary sources.

The quasi-polar orbit and low geomagnetic cut-off experienced by the PAMELA apparatus, combined with its intrinsic ability to measure low momenta, allows phenomena connected with Sun activity and Earth magnetosphere to be investigated.

PAMELA (see Fig. 1) is housed inside a pressurized vessel, $2 \mathrm{~mm}$ thick, and installed on-board of the Resurs-DK1 satellite, following an orbit with altitude varying between 350 and $600 \mathrm{~km}$ and inclination of $70^{\circ}$. PAMELA is continuously taking since July 2006; the mission is foreseen to last until the end of 2011. The core of the PAMELA apparatus is an high-precision magnetic spectrom-

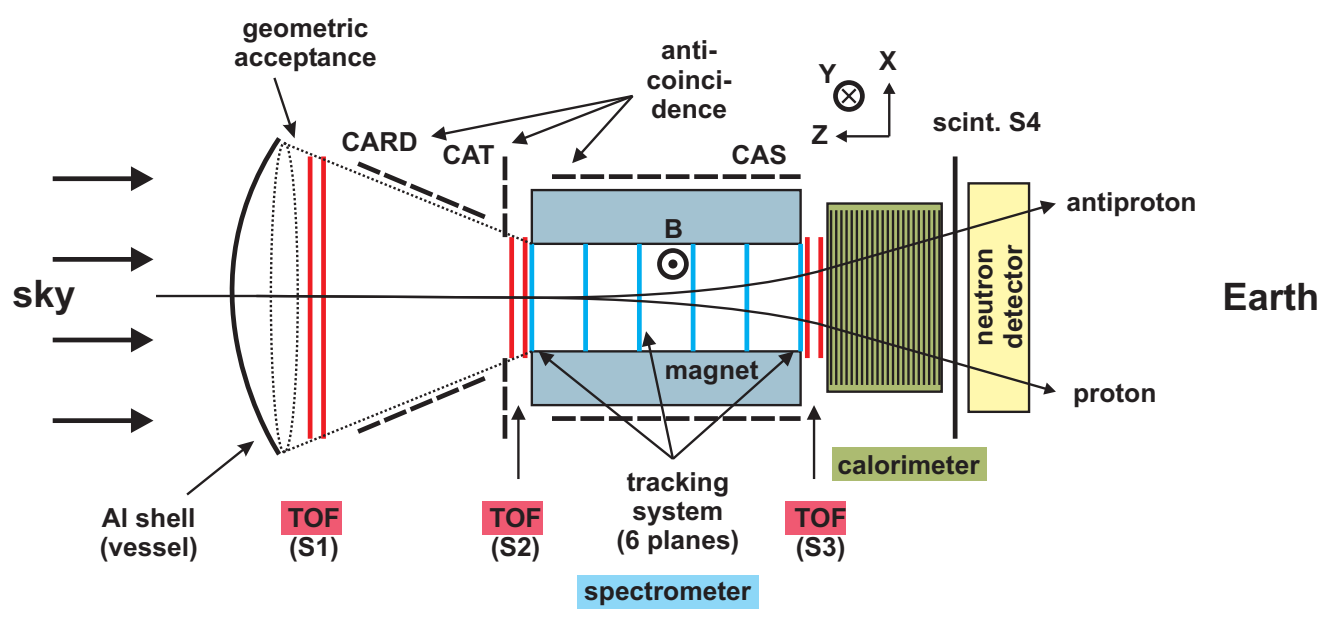

Figure 1: Schematic view of the PAMELA apparatus, with the indication of the main direction of the magnetic field inside the spectrometer cavity. PAMELA is $1.3 \mathrm{~m}$ long and less than $1 \mathrm{~m}$ wide.

eter, with a geometrical factor of $21.6 \mathrm{~cm}^{2} \mathrm{sr}$, and an MDR (Maximum Detectable Rigidity) of the order of $1 \mathrm{TV} / \mathrm{c}$.

The time-of-flight (ToF) system has been designed to provide the main trigger for the experiment and to measure the velocity of the cosmic rays; a time-of-flight resolution better than $300 \mathrm{ps}$ allows $\mathrm{e}^{-} / \mathrm{e}^{+}$to be separated from $\mathrm{p} / \overline{\mathrm{p}}$ up to an energy of $\sim 1 \mathrm{GeV}$. Upward-going (i.e. albedo) particles are rejected with a significance of 60 standard deviations.

The Silicon-Tungsten sampling electromagnetic calorimeter is mainly used to discriminate $\mathrm{e}^{+}$ $\left(\mathrm{e}^{-}\right)$from $\mathrm{p}(\overline{\mathrm{p}})$; by measuring the longitudinal and transverse development of the shower, a very high $\left(\simeq 10^{5}\right)$ rejection power for electromagnetic showers against interacting and non-interacting hadrons could be reached.

An anti-coincidence (AC) system, consisting of plastic scintillators surrounding the magnet's walls, complete the PAMELA apparatus. 


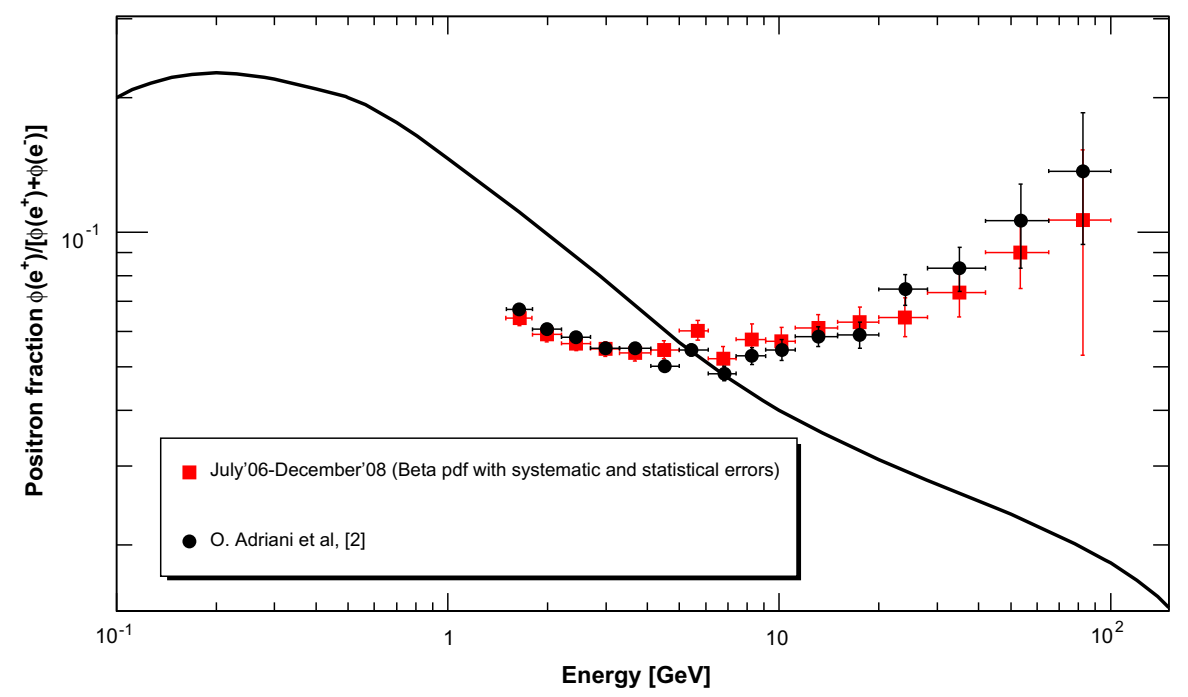

Figure 2: Black dots: the positron fraction measured by PAMELA as published in 2009 [4]. Red dots: the recently published new measurement [2]; the vertical bars include both statistical and systematic errors. Black line: a classical theoretical calculation [5] for pure secondary production of positrons during the propagation of cosmic rays in the galaxy. Figure from Ref. [2].

\section{Positron-to-electron ratio}

The most important aspect to be considered for the measurement of the positron fraction (i.e. the $\mathrm{e}^{+}$flux divided by the sum of $\mathrm{e}^{+}$and $\mathrm{e}^{-}$fluxes) is to keep under control the proton contamination in the positron sample. In our conservative approach, fully described in [2], the residual proton contamination is quantified by using only in-flight data, without any dependence on simulations or beam-test data. A practically pure proton sample is obtained by requiring non-interacting particles in the upper 2 (out of 22) calorimeter modules; the selection cuts for $\mathrm{e}^{+}$are then applied to calorimeter variables evaluated in the "restricted" calorimeter formed by the lower 20 modules. On the other side, $\mathrm{e}^{+}$(with residual $\mathrm{p}$ contamination) and $\mathrm{e}^{-}$samples, with charge sign given by the spectrometer, are selected by using the same calorimeter variables and selection cuts, applied to the completely equivalent "restricted" calorimeter formed by the upper 20 modules.

Studies with particle beams and simulations showed [3] that PAMELA is able to achieve a proton rejection power better than $10^{5}$ up to an energy of $\sim 200 \mathrm{GeV}$, with a positron selection efficiency of $80 \%$, by using simple topological cuts and requiring the match between the total energy release detected in the calorimeter and the momentum given by the spectrometer.

A first positron fraction measurement from PAMELA was presented in 2009, with data collected up to February 2008 (see Ref. [4]); recently, a new paper [2] has been published, containing data up to December 2008 (with an increase of 30\% in statistics) and a better understanding of systematic uncertainties related to the subtraction of $p$ background: the new results are fully consistent with the previous ones, as shown in Fig. 2.

The positron fraction measured by PAMELA is characterized by one order of magnitude improvement in statistics over previous measurements, and it covers the most extended energy range ever achieved by a single apparatus. The positron fraction above $10 \mathrm{GeV}$ is in agreement with previ- 


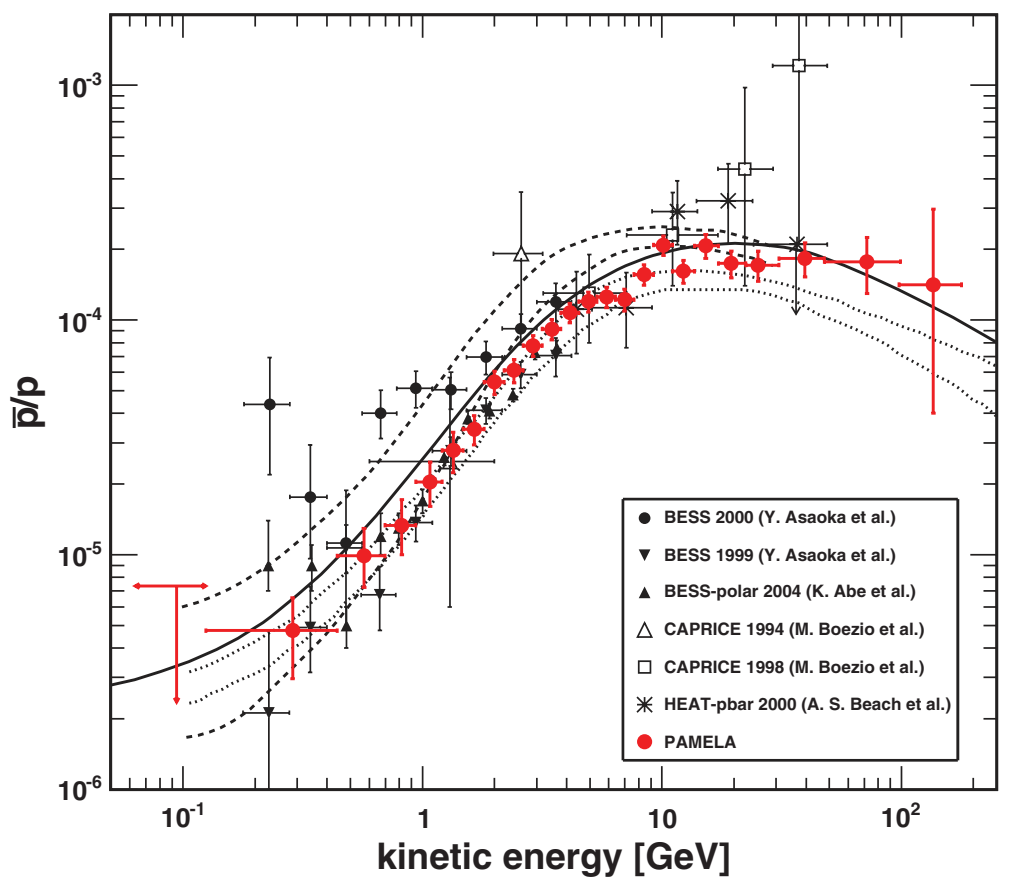

Figure 3: Antiproton/proton flux ratio measured from PAMELA and other recent experiments; the lines represent different theoretical calculations for a pure secondary production of antiprotons during the propagation of cosmic rays in the galaxy. Figure from Ref. [8].

ous measurements, but for the first time it clearly shows a significant increase with energy, that can not be described in the framework of the standard theoretical predictions of secondary production of positrons in the galaxy, even taking into account the large uncertainties in the models involved. Therefore either a significant modification in the theories of cosmic-rays acceleration and propagation is needed, or primary sources like dark-matter annihilation or astrophysical sources (e.g. pulsars), have to be taken into account to describe the PAMELA data. Ref. [6] review in a more complete way some of the possible interpretations of PAMELA positron data.

\section{Antiproton analysis}

The main issue in the antiproton analysis is the background of spillover protons, i.e. protons whose charge sign is wrongly determined because of the uncertainty in the deflection measurement. The spillover contamination is reduced by introducing stronger selection criteria on the quality of the reconstructed trajectory, and by rejecting tracks with low-resolution position measurements related to noisy read-out channels or emission of $\delta$-rays.

The first PAMELA measurement [7] of the $\overline{\mathrm{p}} / \mathrm{p}$ flux ratio, in the kinetic energy range 1.5$100 \mathrm{GeV}$, has been followed by a new publication [8] including more recent data and more refined analysis methods, extending the measurement down to $60 \mathrm{MeV}$ and up to $180 \mathrm{GeV}$ kinetic energies (see Fig. 3).

The measured $\bar{p} / \mathrm{p}$ ratio increases smoothly with kinetic energy up to about $10 \mathrm{GeV}$ and then levels off. The data follow the trend expected from models of secondary production in the galaxy, 
but at the same time these results are precise enough to place strong constraints on theoretical calculations and on possible contributions from exotic sources, e.g. dark-matter particle annihilations. The new publication [8] also contains the first PAMELA measurement of the absolute antiproton flux.

\section{Absolute fluxes of proton and Helium}

Cosmic-ray Hydrogen (mostly protons, with a $\sim 1 \%$ deuterium component) and Helium ( $~$ $10 \%$ of $\mathrm{H}$ ) are overwhelming with respect to other particle species; therefore pure samples of events can be obtained, which are not significantly affected by contamination backgrounds. In fact, this particular measurement of absolute flux is mostly affected by the instrumental systematics in the determination of the selection efficiencies and, above few hundreds $\mathrm{GeV}$, in the spectrometer measurement of the particle rigidity; specifically, possible energy-dependent systematic biases, which can alter the shape of the measured spectra, have to be kept under control.

Given the performances of the PAMELA detectors, the purity of the in-flight collected samples of $\mathrm{H} / \mathrm{He}$ and their high statistics, it has been possible to measure the absolute fluxes of $\mathrm{H}$ and $\mathrm{He}$ over more than three decades of rigidity, up to $\approx 1 \mathrm{TV}$, with an unprecedented total (statistical and systematic) precision of few $\%$. A dedicated paper with the results of this analysis has been prepared [9], presenting the proton and He fluxes in the energy range $1 \mathrm{GeV}-1.2 \mathrm{TeV}$ (1-600 $\mathrm{GeV} / \mathrm{n}$ for $\mathrm{He}$ ); the particle's fluxes have been analyzed both in term of rigidity and kinetic energy per nucleon, converted from the rigidity measured in the tracker and neglecting any contribution from less abundant deuterium and isotopes different from ${ }^{4} \mathrm{He}$.

The PAMELA measurement of $\mathrm{H}$ and He fluxes, covering a very broad energy range and realized with a high precision detector, greatly reducing the systematic errors, will significantly contribute to strongly constrain the theoretical cosmic-ray models, and in particular to reduce the uncertainties on the calculations of the expected secondary antiparticles spectra.

\section{References}

[1] P. Picozza et al., Astropart. Phys. 27, 296 (2007).

[2] O. Adriani et al., Astropart. Phys. 34, 1 (2010).

[3] M. Boezio et al., Astropart. Phys. 26, 111 (2006).

[4] O. Adriani et al., Nature 458, 607 (2009).

[5] I.V. Moskalenko and A.W. Strong, Astrophys. J. 493, 694 (1998).

[6] M. Boezio et al., New J. Phys. 11, 105023 (2009).

[7] O. Adriani et al., Phys. Rev. Lett. 102, 051101 (2009).

[8] O. Adriani et al., Phys. Rev. Lett. 105, 121101 (2010).

[9] O. Adriani et al., PAMELA Measurements of Cosmic-ray Proton and Helium Spectra, Submitted to Science. 